\title{
Generalized and specific anxiety in adolescents following heart transplant
}

\author{
Amanda D. McCormick ${ }^{1}$ (D) | Kurt R. Schumacher ${ }^{1,2}$ | Mary Zamberlan ${ }^{1}$ | Karen Uzark ${ }^{1}$ | \\ Sunkyung $\mathrm{Yu}^{1}$ | Ray Lowery ${ }^{1}$ | Nichole Rottach ${ }^{1}$ | Melissa K. Cousino ${ }^{1,2}$ (C)
}

${ }^{1}$ Department of Pediatrics, Michigan Medicine, Ann Arbor, Michigan

${ }^{2}$ University of Michigan Transplant Center, Ann Arbor, Michigan

\section{Correspondence}

Amanda D. McCormick, Department of Pediatrics, Michigan Medicine, Ann Arbor, MI.

Email: acdelong@med.umich.edu

Funding information

Michigan Congenital Heart Outcomes

Research and Discovery (MCHORD)

\begin{abstract}
Mental health concerns are associated with worse outcomes after adult heart transplant. Illness-specific anxiety is associated with worsened psychological well-being after other solid organ transplants but has never been characterized after pediatric heart transplant. This single-center cross-sectional study aimed to evaluate illnessspecific and generalized anxiety after heart transplantation in adolescents. A novel 12-item PHTF, GAD-7, and the PedsQL were administered. Univariate associations of demographics, clinical features, and medication adherence as measured by immunosuppression standard deviation with the PHTF and GAD-7 scores were evaluated. Internal consistency and validity of the PHTF were examined. In total, 30 patients participated. The most common illness-specific fears were retransplantation, rejection, and more generally post-transplant complications. The PHTF had good internal consistency (Cronbach $\alpha=.88$ ). Construct validity was demonstrated between PHTF and GAD-7 ( $r=.62$ ) and PedsQL ( $r=-.54$ to -.62$) .23 \%$ endorsed moderate to severe generalized anxiety symptoms. More severe symptoms were associated with older age at survey $(P=.03)$, older age at listing $(P=.01)$ and having post-transplant complications $(P=.004)$. Patients with moderate or severe symptoms were more likely to report late immunosuppression doses $(P=.004)$. Illness-specific and generalized anxiety may be prevalent after pediatric heart transplant. Screening for anxiety in adolescents post-transplant may identify those at risk for adverse outcomes including non-adherence. The PHTF is a brief, valid, and reliable instrument identifying illnessspecific anxiety in this population.
\end{abstract}

KEYWORDS

adolescents, generalized anxiety, illness-specific anxiety, pediatric heart transplant

\section{1 | INTRODUCTION}

Despite advancements in medical and surgical management, heart transplantation is the only life-prolonging option for some pediatric

Abbreviations: ECMO, extracorporeal membrane oxygenation; GAD-7, generalized anxiety disorder-7 scale; PedsQL, pediatric quality of life inventory; PHTF, post heart transplant fears questionnaire; VAD, Ventricular assist device. patients with end-stage heart disease. According to data from the Pediatric Heart Transplant Study, the numbers of both listed and transplanted pediatric patients are slowly increasing, with improving post-transplant survival. ${ }^{1}$ However, the risk of morbidity and death remain. For many pediatric patients and their caregivers, life postheart transplantation results in improved physical functioning, but also involves the management of a new chronic condition and the potential for complications such as infection, rejection, renal failure, 
and malignancy. ${ }^{1}$ In addition, transplant recipients face the inevitable risk of coronary allograft vasculopathy which increases with time ${ }^{2}$ resulting ultimately in reoccurrence of heart failure symptoms and potential for retransplantation.

Transplantation also seeks to improve the health-related quality of life for pediatric patients with end-stage heart disease; however, a subset remains at risk for impaired psychological well-being following transplant, ${ }^{3}$ as well as impaired health-related quality of life, neuropsychological outcomes, and family function as compared to non-transplant recipients. ${ }^{4}$ While mental health disorders are well studied in adult patients after heart transplantation, ${ }^{5-7}$ less is known about the long-term mental health of pediatric heart transplant recipients. ${ }^{8-10}$

Limited studies suggest that pediatric heart transplant patients may have increased risk of psychological disorders when compared to the general population. One study found that a quarter or greater of pediatric heart transplant recipients are at risk for emotional adjustment issues immediately following transplant. ${ }^{11}$ Previous research suggests that approximately a third of patients continue to experience significant psychosocial distress in the years following heart transplantation, ${ }^{12}$ including symptoms of post-traumatic stress disorder ${ }^{13}$ and emotional and behavioral disorders. ${ }^{14}$ It is possible that mental health concerns are worse in patients with a more complicated clinical course, given prior studies in adult heart transplant patients associating anxiety and depression and hospital length of stay, ${ }^{7}$ although this association has not been explored in the pediatric population.

The causes of impaired psychological well-being after heart transplant are not well understood. It is possible that illness-specific anxiety about post-transplant complications contributes to worse health-related quality of life post-transplant. Fear of rejection following solid organ transplants has been identified in an adult population, ${ }^{15,16}$ and intrusive thoughts about graft rejection have been shown to contribute to poor psychological well-being after lung transplantation. ${ }^{17}$ Across other chronic illness populations, there is growing evidence for the presence of illness-specific anxiety and fears related to disease progression or recurrence, ${ }^{18-20}$ distinct from generalized anxiety disorder. ${ }^{21}$ Importantly, illness-specific anxiety has been associated with generalized anxiety, depression, and increased healthcare utilization in other adult chronic illness populations. $^{22}$

In adult transplant patients, mental health concerns such as generalized anxiety and depression are also associated with increased risk of graft loss and death. ${ }^{23}$ One factor contributing to poor outcomes in adult recipients may be the association between poor mental health and non-adherence to medication regimen. ${ }^{24,25}$ Mental health concerns in pediatric transplant patients may similarly contribute to non-adherence, which in turn increases morbidity and mortality. ${ }^{26}$ Illness-specific anxiety as a possible contributor has yet to be studied in pediatric transplant recipients. Adult transplant and other chronic illness literatures underscore the importance of examining illness-specific anxiety in pediatric transplant recipients as it may contribute to poorer mental health and subsequent risk of adverse health outcomes.
The aims of the current study were (a) to develop and validate a novel measure to assess illness-specific anxiety in adolescents after heart transplantation, (b) to characterize the prevalence and scope of illness-specific anxiety and generalized anxiety in adolescents after heart transplant, and (c) to evaluate for association between illness-specific anxiety and generalized anxiety symptoms following heart transplant with health experiences prior to and after transplantation. It was hypothesized that illness-specific and generalized anxiety are both prevalent among adolescent patients post-heart transplantation. It was also hypothesized that illness-specific and generalized anxiety are associated with complications in clinical course both before and after transplantation and worse medication adherence.

\section{2 | METHODS}

\section{1 | Procedures and recruitment}

This single-center cross-sectional study included all patients with a history of heart transplantation greater than 12 years of age followed within a pediatric heart transplant program who gave written informed consent or written informed assent with parent or guardian written informed consent if $<18$ years of age. Patients were excluded if they were non-English speaking or unable to complete the questionnaires due to cognitive/developmental impairment. Two patients were excluded as they were assessed to be nearing end of life by the clinical team, and thus likely to be inappropriate for the present study.

The study was approved by the Institutional Review Board. Heart transplant recipients were approached by the principal investigator or the research coordinator at the time of a routine outpatient follow-up visit and enrolled patients independently completed all measures detailed below. Demographic characteristics and information on clinical course were obtained by review of the electronic health record.

\section{2 | Adherence}

Immunosuppression troughs were recorded, and a standard deviation of trough levels was calculated as a surrogate for medication adherence. $^{27,28}$ Additionally, patients' self-report of adherence was obtained by review of clinic notes on the day of the surveys. Specifically, patients were asked "how many missed or late immunosuppression doses have you had in the last week?".

\section{3 | Existing measures}

The GAD-7 is a self-report instrument designed for adults consisting of seven items measuring generalized anxiety. Items have four response options increasing in frequency, and scores are summed with higher scores reflecting higher severity of symptoms. ${ }^{29}$ The GAD-7 has been validated for detection of anxiety symptoms in adolescents with generalized anxiety disorder ${ }^{30}$ and adult heart transplant recipients. ${ }^{31}$ 
The PedsQL 4.0 Generic Core Scale is a 23-item instrument that assesses physical, emotional, social, and school functioning. The PedsQL 3.0 Cardiac Module is a 27-item instrument that assesses symptom burdens, perceived physical appearance, treatment anxiety, cognitive problems, and communication. All items are scored on a 5-point Likert scale. Items are reverse-scored and linearly transformed to a 100-point scale such that higher scores indicate a better health-related quality of life. The PedsQL Generic Core Scale is used to create a psychosocial health summary score, computed as the sum of the items divided by the number of items in the emotional, social, and school functioning scales. ${ }^{32}$ Both the PedsQL 4.0 Generic Core Scales and PedsQL 3.0 Cardiac Module have been utilized in pediatric heart transplant recipients. ${ }^{3}$

\subsection{Statistical analyses}

Data are reported as means \pm standard deviations or median with interquartile range (IQR) for continuous variables and frequencies with percentages for categorical variables. Total GAD-7 scores were considered as both continuous and binary variables with patients dichotomized into two groups, those reporting moderate or severe anxiety symptoms (GAD-7 score $\geq 10$ ) and those reporting minimal or mild anxiety symptoms (GAD-7 score 0-9). Associations of patient demographics, pretransplant course, transplant characteristics, and post-transplant course with GAD-7 scores (both continuous and binary variables) were assessed using Fisher's exact test, Spearman correlation coefficient, Wilcoxon rank sum test, or Kruskal-Wallis test, as appropriate. Total (PHTF, see below) scores and individual PHTF item score were examined as a 5-point Likert scale, as well as linearly transformed a 100-point scale such that higher scores indicating more anxiety. Associations between PHTF scores and patient demographics, pretransplant course, transplant characteristics, and post-transplant course were assessed using a two-sample $t$ test or analysis of variance for categorical variables, and Spearman correlation coefficient for continuous variables. All analyses were performed using SAS Version 9.4 (SAS Institute Inc), with statistical significance set at a $P$-value $<.05$ using a two-sided test.

\section{I PHTF: MEASURE DEVELOPMENT AND VALIDATION}

\section{1 | Measure development}

The novel PHTF questionnaire was developed after discussion with an experienced survey researcher. Validated measures of illness-specific anxiety in other chronic disease states were reviewed. ${ }^{16-20,33}$ Items from published measures that were applicable to the post-heart transplant experience were selected or adapted as appropriate, and nine items were selected with responses on a 5-point Likert scale ranging from "never" (1) to "always" (5). An additional three items were included inquiring about understanding of complications of heart transplantation and discussing worries about complications with loved ones and healthcare team members with responses on a 5-point Likert scale ranging from "strongly disagree" (1) to "strongly agree" (5). All metric items were discussed with experts in medical management of pediatric heart transplantation as well as psychological care post pediatric heart transplantation. The PHTF was reviewed by two adolescent heart transplant patients for comprehension and context prior to conducting the study. The PHTF questionnaire items are included in Table 3.

\section{2 | Psychometric properties of PHTF}

The psychometric properties of the PHTF were examined. Scale internal consistency reliability was determined by Cronbach's coefficient alpha $(\alpha)$. To establish construct validity, intercorrelations between the PHTF scores and total GAD-7 score, PedsQL 4.0 Generic Core Scale Psychosocial summary score, and PedsQL Cardiac Module Treatment Anxiety subscale score were performed using Pearson or Spearman correlation coefficient.

The internal consistency of the PHTF was good (Cronbach $\alpha=.88)$. Construct validity was also demonstrated with medium to high $r$ values between PHTF total score, GAD-7 total score $(r=.62)$ and the PedsQL Psychosocial summary score $(r=-.54)$ and Cardiac Module Treatment Anxiety subscale score ( $r=-.62$; Table 1$)$.

\section{RESULTS}

\section{1 | Patient characteristics}

Table 2 summarizes patient demographic and clinical characteristics. Of 31 eligible patients, only one patient declined participation in the study yielding a participation rate of $97 \%$. Most patients were male (73.3\%) and Caucasian (80\%) with a median age of 17.5 years. There was about equal representation of pretransplant diagnosis of congenital heart disease and cardiomyopathy. Median time from transplant at the time of survey was 8.0 years. Thirty percent of patients had experienced a pretransplant complication including cardiac arrest, stroke, need for (ECMO) and/ or need for (VAD). Thirty percent of patients experienced a posttransplant clinical complication including cardiac arrest, stroke, need for ECMO and/or need for dialysis. Thirty percent of patients had coronary vasculopathy, $33 \%$ had at least one episode of graft rejection.

\section{2 | Adherence}

Most patients were on tacrolimus (93.3\%) with a median immunosuppression standard deviation of $1.56(43 \% \geq 2)$. Twenty percent of 
TAB LE 1 PHTF validation: correlation between overall anxiety score from PHTF questionnaires and other validated measures (GAD-7, PedsQL)

\begin{tabular}{|lll|}
\hline & \multicolumn{2}{l}{$l$} \\
\hline GAD-7 total score & 0.62 & .0003 \\
\hline PedsQL generic core total score & -0.56 & .001 \\
\hline Physical functioning subscale & -0.48 & .01 \\
\hline Psychosocial Summary subscale & -0.54 & .002 \\
\hline Emotional functioning subscale & -0.71 & $<.0001$ \\
\hline Social functioning subscale & -0.32 & .09 \\
\hline School functioning subscale & -0.18 & .36 \\
\hline PedsQL cardiac module & & \\
\hline Heart problems and treatment subscale & -0.44 & .02 \\
\hline Treatment II subscale (if taking & -0.49 & .01 \\
\hline medications) & & \\
\hline Perceived physical appearance subscale & -0.71 & $<.0001$ \\
\hline Treatment anxiety subscale & -0.62 & .0003 \\
\hline Cognitive problems subscale & -0.28 & .13 \\
\hline Communication subscale & -0.67 & $<.0001$ \\
\hline
\end{tabular}

Data are presented as $r$, Pearson or Spearman correlation coefficient ( $P$-value).

patients reported at least one late dose of immunosuppression in the last week.

\section{3 | Illness-specific anxiety}

There was a significant association between greater PHTF score and greater GAD-7 score or more severe generalized anxiety $(r=.62, P=.0003)$. There also was a significant association between greater PHTF score with a lower PedsQL total score $(r=-.56, P=.001)$ and PedsQL Psychosocial subscale score $(r=-.54, P=.002)$, indicating worse health-related quality of life and psychological well-being.

There were three items within the PHTF that a notable percentage of patients reported worrying about "often" or "always" (Table 3). These included worrying about rejection while waiting for biopsy results (26.7\%), worrying about needing another transplant (20\%), and serious post-transplant complications (20\%). When specifically asked about their worries, a notable percentage of patients reported that they were not discussing fears about long-term complications with their family or providers. About a quarter of patients chose that they "disagree" or "strongly disagree" with the statement that they discuss their worries about long-term complications of heart transplantation with their loved ones (23.3\%) and healthcare team (13.3\%), and 10\% disagreed that they had a good understanding of the complications of heart transplant.

Higher overall illness-specific anxiety was associated with female gender (mean PHTF score $46.2 \pm 14.0$ vs $23.8 \pm 22.7$ in male, $P=.01$ ). Individual PHTF item analysis revealed a significant
TABLE 2 Patient demographic, transplant, and post-transplant characteristics $(\mathrm{N}=30)$

\begin{tabular}{|c|c|}
\hline & $\begin{array}{l}\mathrm{N}(\%) \text { or } \\
\text { Median } \\
\text { (interquartile } \\
\text { range) }\end{array}$ \\
\hline Male gender & $22(73.3)$ \\
\hline Age at survey, years & $17.5(15.6-19.2)$ \\
\hline Time from transplant to survey, years & $8.0(4.0-14.6)$ \\
\hline Caucasian race & $24(80.0)$ \\
\hline \multicolumn{2}{|l|}{ Diagnosis } \\
\hline Cardiomyopathy & $15(50.0)$ \\
\hline Congenital heart disease & $14(46.7)$ \\
\hline Other (Familial hyperlipidemia) & $1(3.3)$ \\
\hline Pretransplant & $9(30.0)$ \\
\hline History of cardiac arrest & $3(10.0)$ \\
\hline History of stroke & $1(3.3)$ \\
\hline Need for ECMO & $5(16.7)$ \\
\hline Need for VAD & $2(6.7)$ \\
\hline Pretransplant ICD & $1(3.3)$ \\
\hline Age at listing, years $(N=28)$ & $7.6(3.3-13.4)$ \\
\hline \multicolumn{2}{|l|}{ Listing status } \\
\hline $1 \mathrm{~A}$ & $15(50.0)$ \\
\hline $1 \mathrm{~B}$ & $3(10.0)$ \\
\hline 2 & $11(36.7)$ \\
\hline Unknown & $1(3.3)$ \\
\hline Waiting time, days $(\mathrm{N}=28)$ & $55(22.5-188)$ \\
\hline Age at transplant, years & $8.3(3.1-14.3)$ \\
\hline \multicolumn{2}{|l|}{ NYHA class } \\
\hline 1 & $1(3.3)$ \\
\hline II & $10(33.3)$ \\
\hline III & $14(46.7)$ \\
\hline IV & $4(13.3)$ \\
\hline Unknown & $1(3.3)$ \\
\hline $\begin{array}{l}\text { Hospital length of stay since Transplant, days } \\
(N=29)\end{array}$ & $14(12-22)$ \\
\hline Post-transplant complication & $9(30.0)$ \\
\hline Cardiac arrest & $2(6.7)$ \\
\hline Stroke & $3(10.0)$ \\
\hline Need for ECMO & $5(16.7)$ \\
\hline Need for dialysis & $4(13.3)$ \\
\hline Coronary vasculopathy & $9(30.0)$ \\
\hline $\begin{array}{l}\text { Post-transplant lymphoproliferative disorder } \\
\text { (PTLD) }\end{array}$ & $4(13.3)$ \\
\hline Antibody mediated rejection (AMR) & $3(10.0)$ \\
\hline Rejection episodes & $10(33.3)$ \\
\hline Unplanned hospitalization in the past year & $7(23.3)$ \\
\hline
\end{tabular}

association between immunosuppression standard deviation $\geq 2$ and increased patient worry about rejection while waiting for 
TABLE 3 PHTF questionnaires $\mathrm{N}=30$

\begin{tabular}{|c|c|c|c|c|c|c|c|c|}
\hline & Never & Rarely & Sometimes & \multicolumn{2}{|l|}{ Often } & Always & \multicolumn{2}{|c|}{ Not reported } \\
\hline I worry about needing another transplant & $8(26.7)$ & $7(23.3)$ & $9(30.0)$ & \multicolumn{2}{|l|}{$2(6.7)$} & $4(13.3)$ & \multicolumn{2}{|l|}{-} \\
\hline I am nervous prior to doctor's appointments & $15(50.0)$ & $7(23.3)$ & $4(13.3)$ & \multicolumn{2}{|l|}{$2(6.7)$} & $2(6.7)$ & \multicolumn{2}{|l|}{-} \\
\hline $\begin{array}{l}\text { I worry about rejection of my heart waiting } \\
\text { for results from my biopsies }\end{array}$ & $9(30.0)$ & $7(23.3)$ & $6(20.0)$ & \multicolumn{2}{|l|}{4 (13.3) } & 4 (13.3) & \multicolumn{2}{|l|}{-} \\
\hline $\begin{array}{l}\text { I worry about missing school or work due to } \\
\text { complications of my heart transplant }\end{array}$ & $14(46.7)$ & $7(23.3)$ & $3(10.0)$ & \multicolumn{2}{|l|}{$5(16.7)$} & $0(0.0)$ & \multicolumn{2}{|l|}{$1(3.3)$} \\
\hline $\begin{array}{l}\text { I worry about serious post-transplant } \\
\text { complications }\end{array}$ & $10(33.3)$ & $9(30.0)$ & $5(16.7)$ & \multicolumn{2}{|l|}{$3(10.0)$} & $3(10.0)$ & \multicolumn{2}{|l|}{-} \\
\hline \multirow{2}{*}{$\begin{array}{l}\text { I don't enjoy life because I am worried about } \\
\text { complications from my heart transplant }\end{array}$} & $18(60.0)$ & $4(13.3)$ & $7(23.3)$ & \multicolumn{2}{|l|}{$0(0.0)$} & $1(3.3)$ & \multicolumn{2}{|l|}{-} \\
\hline & & Strongly disagree & Disagree & Neutral & Agree & Strongly & agree & \\
\hline \multicolumn{9}{|c|}{ How much do you agree with the following statements } \\
\hline \multicolumn{2}{|c|}{$\begin{array}{l}\text { I have a good understanding of the long-term complica- } \\
\text { tions of heart transplantation }\end{array}$} & $0(0.0)$ & $3(10.0)$ & $7(23.3)$ & $7(23.3)$ & $13(43.3)$ & & - \\
\hline \multicolumn{2}{|c|}{$\begin{array}{l}\text { I discuss my worries about long-term complications of } \\
\text { heart transplantation with my loved ones }\end{array}$} & $5(16.7)$ & $2(6.7)$ & $6(20.0)$ & $8(26.7)$ & $9(30.0)$ & & - \\
\hline
\end{tabular}

Data are presented as N (\%).

biopsy results (mean $61.5 \pm 32.1$ vs $41.7 \pm 25.7 \mathrm{SD}<2, P=.04$ ). There were no other associations of demographic or clinical features with PHTF total score, including age at survey $(r=.28$, $P=.13$ ) and post-transplant complications including cardiac arrest, stroke, ECMO, or dialysis (mean $38.6 \pm 21.6$ vs $24.8 \pm 22.7$ without complications, $P=.14$ ).

\section{4 | Generalized anxiety}

Nearly, a quarter of the patients (23.3\%) reported moderate to severe anxiety symptoms (GAD-7 score $\geq 10$ ), Table 4). Of the individual items in the GAD-7, patients were most likely to report "becoming annoyed or irritable" (43.3\%), "feeling afraid as if something awful might happen" (30\%), "feeling nervous, anxious, or on edge" (26.7\%), and "worrying too much about different things" (26.7\%) over half the days or nearly every day.

Higher GAD-7 scores or more severe generalized anxiety symptoms were significantly associated with older age at the time of the survey $(r=.41, P=.03)$, older age at transplant listing $(r=.46, P=.01)$, and any post-transplant complication (cardiac arrest, stroke, need for
ECMO and/or need for dialysis) (median GAD-7 score 10 vs 1.5 without complication, $P=.004$ ). Each post-transplant complication was evaluated individually, and both stroke (median 15 vs $3.5, P=.03$ ) and need for ECMO (median 10 vs $2.5, P=.01$ ) were significantly associated with more severe generalized anxiety symptoms. There were no significant associations with any other patient demographic criteria, pretransplant complications, history of rejection, history of coronary vasculopathy, ED visits or hospitalizations, or immunosuppression trough standard deviation.

Notably, moderate or severe generalized anxiety symptoms were significantly associated with patients reporting late (>2 hours after dose time) or missed immunosuppression doses within the past 2 weeks $(57.1 \%$ vs. $8.7 \%, P=.004)$ during clinical interview, compared to those that expressed minimal or mild generalized anxiety symptoms.

\section{5 | DISCUSSION}

To our knowledge, this is the first study to evaluate illness-specific anxiety in pediatric patients after heart transplantation. In addition, the study may provide further insight into the prevalence 
TABLE 4 GAD-7 Scale $(\mathrm{N}=30)$

\begin{tabular}{|c|c|c|c|c|c|}
\hline & & Not at all sure & Several days & $\begin{array}{l}\text { Over half the } \\
\text { days }\end{array}$ & Nearly every day \\
\hline \multicolumn{6}{|c|}{ Over the last $2 \mathrm{wk}$, how often have you been bothered by the following problems } \\
\hline \multicolumn{2}{|c|}{ Not being able to stop or control worrying } & $20(66.7)$ & $4(13.3)$ & $3(10.0)$ & $3(10.0)$ \\
\hline \multicolumn{2}{|c|}{ Worrying too much about different things } & $15(50.0)$ & $7(23.3)$ & $2(6.7)$ & $6(20.0)$ \\
\hline \multicolumn{2}{|l|}{ Being so restless that it's hard to sit still } & $19(63.3)$ & $6(20.0)$ & $3(10.0)$ & $2(6.7)$ \\
\hline \multicolumn{2}{|l|}{ Becoming easily annoyed or irritable } & $12(40.0)$ & $5(16.7)$ & $6(20.0)$ & $7(23.3)$ \\
\hline \multicolumn{2}{|c|}{ Feeling afraid as if something awful might happen } & $15(50.0)$ & $6(20.0)$ & $4(13.3)$ & $5(16.7)$ \\
\hline \multicolumn{3}{|l|}{ Total score, median (IQR) } & $5(0-9)$ & & \\
\hline \multirow{2}{*}{\multicolumn{2}{|c|}{ Severe (score 15 and higher) }} & & $3(10.0)$ & & \\
\hline & & Not difficult at all & Somewhat difficult & Very difficult & Extremely difficult \\
\hline $\begin{array}{l}\text { If you checked off any problems, how } \\
\text { difficult have these made it for you to } \\
\text { do your work, take care of things at } \\
\text { home, or get along with other people }\end{array}$ & $N=20$ & $3 / 20(15.0)$ & $14 / 20(70.0)$ & $2 / 20(10.0)$ & $0 / 20(0.0)$ \\
\hline
\end{tabular}

Data are presented as $\mathrm{N}(\%)$ unless otherwise indicated.

of generalized anxiety in this population. Our results suggest that illness-specific anxiety, as well as generalized anxiety, may be prevalent in adolescent and young adult patients after heart transplant.

Nearly a quarter of patients in the study reported moderate to severe generalized anxiety symptoms, which is almost five times the prevalence of generalized anxiety in the healthy adolescent population. ${ }^{34}$ Chronic illness in adolescents is a risk factor for anxiety disorders ${ }^{35}$ which may explain the higher prevalence in the post-transplant population. Additionally, older patient age at the time of study and at listing was associated with more severe generalized anxiety symptoms. As such, it may be important to target this older population of pediatric heart transplant recipients for screening, as they may be more likely to require additional mental health intervention. Interestingly, generalized anxiety symptoms were not significantly associated with patient gender in the current study, whereas generalized anxiety is more prevalent in females in the general population. ${ }^{36}$ It may be that adolescent patients after heart transplant are disproportionately affected by generalized anxiety symptoms, regardless of gender effects. Alternatively, gender effects may not have been observed due to the study's $73 \%$ male predominance.

In addition to generalized anxiety, illness-specific anxiety was prevalent among the study sample across all ages with $20 \%-30 \%$ of patients endorsing fears related to need for retransplantation, graft rejection, or complications. Furthermore, unlike with generalized anxiety, illness-specific anxiety was associated with female gender, which may identify female patients as a population at increased need for screening. Notably, illness-specific anxiety was not associated with any pre- or post-heart transplant complications. Thus, it would seem that a notable percentage of adolescents with heart transplants will have worries about complications regardless of their clinical course. Finally, results suggested that adolescents may not be discussing their fears with their families or their loved ones, further underscoring the importance of screening.

Findings also suggested that the presence of generalized anxiety symptoms is associated with late immunosuppression doses per patient report during clinical interview. This is consistent with previous studies that have shown that mental health disorders are associated with non-adherence in the adolescent population. ${ }^{26}$ Similarly, a study of adolescent liver transplant recipients found that patients with greater immunosuppression standard deviation reported worse mental health than those with less variation of immunosuppressant trough levels. ${ }^{37}$ Additionally, patients in the current study who had more variability in their immunosuppression trough levels were more likely to endorse illness-specific worries about graft rejection. Another study in adolescent transplant patients associated non-adherence with both symptoms of depression and worries related to their illness. ${ }^{38}$ Whether having anxiety drives non-adherence or non-adherence contributes to further anxiety is currently unclear, however, it is fair to conclude that adolescent patients identified with generalized or illness-specific anxiety may require more support to ensure medication adherence and improve their long-term health outcomes. 
Like many pediatric transplant studies, this single-center sample size is small. The study population also only focused on adolescent aged pediatric transplant patients. Development of more simplistic metrics of illness-specific anxiety will be needed for studies with younger patients. Larger, multi-center studies would also be valuable for evaluating the prevalence of anxiety across the entire pediatric heart transplant population as well as examining associations with longitudinal health outcomes including morbidity and mortality. Additionally, the PHTF is a novel measure, and not validated for transplant-specific anxiety outside of this study. The current study did not evaluate the presence of other mental health disorders, such as depression. Although anxiety and depression often overlap, prevalence and impact on outcomes may differ. The current study was also limited by the lack of reliable strategies for evaluating non-adherence. While immunosuppression standard deviation has been used in prior studies, this is a surrogate measure of adherence which may be impacted by additional factors such as drug absorption or medication interactions. ${ }^{27,28}$ Furthermore, self-reported medication adherence may not always be reliable, especially in the adolescent population.

Despite these limitations, this study suggests that adolescent heart transplant patients are likely to benefit from screening for illness-specific and generalized anxiety symptoms with brief, validated instruments. Identification of patients with illness-specific anxiety and generalized anxiety may identify those at risk for medication non-adherence and adverse health outcomes. While there are no formal recommendations for psychosocial care in the pediatric heart transplant population, the $\mathbf{2 0 1 0}$ International Society for Heart and Lung Transplantation Guidelines for care of heart transplant recipients recommend that each heart transplant team have a psychologist and that patients be regularly screened for depression. ${ }^{39}$ Many pediatric centers do not have psychological support readily available, ${ }^{12}$ particularly in outpatient clinic settings. The GAD-7 and PHTF are short and easy to use tools which can be used by multidisciplinary healthcare providers to screen and to identify transplant recipients in need of evaluation and formal psychological treatment.

\section{ACKNOWLEDGMENTS}

The authors would like to acknowledge the Michigan Congenital Heart Outcomes Research and Discovery (MCHORD) group at the University of Michigan Congenital Heart Center, for their support in the development and funding of this project. The authors have no financial disclosures or industry relationships pertinent to this work to report.

\section{AUTHORS' CONTRIBUTIONS}

Amanda D. McCormick: Involved in concept/design, data collection, data analysis/interpretation, statistics, drafting the article, and approval of the article; Kurt R. Schumacher, Melissa K. Cousino, and Karen Uzark: Involved in concept/design, data/analysis/interpretation, critical revision of the article, and approval of the article; Sunkyung Yu and Ray Lowery: Involved in data collection, data analysis/interpretation, statistics, critical revision of the article, and approval of the article; Mary Zamberlan and Nichole Rottach: Collected data, critically revised the article, and approved the article.

\section{ORCID}

Amanda D. McCormick iD https://orcid. org/0000-0002-4874-854X

Melissa K. Cousino (iD https://orcid.org/0000-0002-0041-0830

\section{REFERENCES}

1. Dipchand Al, Kirk R, Mahle WT, et al. Ten yr of pediatric heart transplantation: a report from the pediatric heart transplant study. Pediatr Transplant. 2013;17(2):99-111.

2. Dipchand AI, Edwards LB, Kucheryavaya AY, et al. The registry of the international society for heart and lung transplantation: seventeenth official pediatric heart transplantation report-2014; focus theme: retransplantation. J Heart Lung Transplant. 2014;33(10):985-995.

3. Uzark K, Griffin L, Rodriguez R, et al. Quality of life in pediatric heart transplant recipients: a comparison with children with and without heart disease. J Heart Lung Transplant. 2012;31(6):571-578.

4. Fredericks EM, Zelikovsky N, Aujoulat I, Hames A, Wray J. Post-transplant adjustment-the later years. Pediatr Transplant. 2014;18(7):675-688.

5. Pudlo R, Piegza M, Zakliczyński M, Zembala M. The occurence of mood and anxiety disorders in heart transplant recipients. Transpl Proc. 2009;41(8):3214-3218.

6. Dew MA, DiMartini AF. Psychological disorders and distress after adult cardiothoracic transplantation. J Cardiovasc Nurs. 2005;20(5 Suppl):S51-66

7. Dew MA, Kormos RL, DiMartini AF, et al. Prevalence and risk of depression and anxiety-related disorders during the first three years after heart transplantation. Psychosomatics. 2001;42(4):300-313.

8. Wray J, Radley-Smith R. Depression in pediatric patients before and 1 year after heart or heart-lung transplantation. J Heart Lung Transplant. 2004;23(9):1103-1110.

9. Wray J, Radley-Smith R. Longitudinal assessment of psychological functioning in children after heart or heart-lung transplantation. J Heart Lung Transplant. 2006;25(3):345-352.

10. Wray J, Radley-Smith R. Prospective psychological evaluation of pediatric heart and heart-lung recipients. J Pediatr Psychol. 2007;32(2):217-222.

11. Demaso DR, Douglas Kelley S, Bastardi H, O'Brien P, Blume ED. The longitudinal impact of psychological functioning, medical severity, and family functioning in pediatric heart transplantation. $J$ Heart Lung Transplant. 2004;23(4):473-480.

12. Cousino MK, Schumacher KR, Rea KE, et al. Psychosocial functioning in pediatric heart transplant recipients and their families. Pediatr Transplant. 2018;22(2):e13110.

13. Farley LM, DeMaso DR, D'Angelo E, et al. Parenting stress and parental post-traumatic stress disorder in families after pediatric heart transplantation. J Heart Lung Transplant. 2007;26(2):120-126.

14. Todaro JF, Fennell EB, Sears SF, Rodrigue JR, Roche AK. Review: cognitive and psychological outcomes in pediatric heart transplantation. J Pediatr Psychol. 2000;25(8):567-576.

15. Jamieson NJ, Hanson CS, Josephson MA, et al. Motivations, challenges, and attitudes to self-management in kidney transplant recipients: a systematic review of qualitative studies. Am J Kidney Dis. 2016;67(3):461-478.

16. Nilsson M, Forsberg A, Bäckman L, et al. The perceived threat of the risk for graft rejection and health-related quality of life among organ transplant recipients. J Clin Nurs. 2011;20(1-2):274-282.

17. Forsberg A, Nilsson M, Jakobsson S, Lennerling A, Kisch A Fear of graft rejection 1-5 years after lung transplantation-a nationwide cohort study. Nurs Open. 2018;5(4):484-490. 
18. Lee-Jones C, Humphris G, Dixon R, Bebbington Hatcher M. Fear of cancer recurrence-a literature review and proposed cognitive formulation to explain exacerbation of recurrence fears. Psychooncology. 1997;6(2):95-105.

19. Herschbach P, Berg P, Dankert A, et al. Fear of progression in chronic diseases: psychometric properties of the fear of progression questionnaire. J Psychosom Res. 2005;58(6):505-511.

20. Thewes B, Butow P, Zachariae R, et al. Fear of cancer recurrence: a systematic literature review of self-report measures. Psychooncology. 2012;21(6):571-587.

21. Dinkel A, Kremsreiter K, Marten-Mittag B, Lahmann C. Comorbidity of fear of progression and anxiety disorders in cancer patients. Gen Hosp Psychiatry. 2014;36(6):613-619.

22. Lebel S, Tomei C, Feldstain A, Beattie S, McCallum M. Does fear of cancer recurrence predict cancer survivors' health care use? Support Care Cancer. 2013;21(3):901-906.

23. Dew MA, Rosenberger EM, Myaskovsky L, et al. Depression and anxiety as risk factors for morbidity and mortality after organ transplantation: a systematic review and meta-analysis. Transplantation. 2015;100(5):988-1003.

24. Dew M, Roth LH, Thompson ME, Kormos RL, Griffith BP. Medical compliance and its predictors in the first year after heart-transplantation. J Heart Lung Transplant. 1996;15(6):631-645.

25. Dew M, Kormos RL, Roth LH, Murali S, DiMartini A, Griffith BP. Early post-transplant medical compliance and mental health predict physical morbidity and mortality one to three years after heart transplantation. J Heart Lung Transplant. 1999;18(6):549-562.

26. Dobbels F, Damme-Lombaert RV, Vanhaecke J, Geest SD. Growing pains: non-adherence with the immunosuppressive regimen in adolescent transplant recipients. Pediatr Transplant. 2005;9(3):381-390.

27. Christina S, Annunziato RA, Schiano TD, et al. Medication level variability index predicts rejection, possibly due to nonadherence, in adult liver transplant recipients. Liver Transpl. 2014;20(10):1168-1177.

28. Shemesh E, Bucuvalas JC, Anand R, et al. The medication level variability index (MLVI) predicts poor liver transplant outcomes: a prospective multi-site study. Am J Transplant. 2017;17(10):2668-2678.

29. Spitzer RL, Kroenke K, Williams JBW, Löwe B. A brief measure for assessing generalized anxiety disorder. Arch Intern Med. 2006;166(10):1092.

30. Mossman SA, Luft MJ, Schroeder HK, et al. The generalized anxiety disorder 7-item scale in adolescents with generalized anxiety disorder: Signal detection and validation. Ann Clin Psychiatry. 2017;29(4):227-234A.

31. Conway A, Sheridan J, Maddicks-Law J, et al. Accuracy of anxiety and depression screening tools in heart transplant recipients. Appl Nurs Res. 2016;32:177-181.

32. Uzark K, Jones K, Slusher J, Limbers CA, Burwinkle TM, Varni JW. Quality of life in children with heart disease as perceived by children and parents. Pediatrics. 2008;121(5):e1060-e1067.

33. Simard S, Thewes B, Humphris $G$, et al. Fear of cancer recurrence in adult cancer survivors: a systematic review of quantitative studies. J Cancer Surviv. 2013;7(3):300-322.

34. Burstein M, Beesdo-Baum K, He JP, Merikangas KR. Threshold and subthreshold generalized anxiety disorder among US adolescents: prevalence, sociodemographic, and clinical characteristics. Psychol Med. 2014;44(11):2351-2362.

35. Pao M, Bosk A. Anxiety in medically ill children/adolescents. Depress Anxiety. 2011;28(1):40-49.

36. Vesga-Lopez O, Schneier FR, Wang S, et al. Gender differences in generalized anxiety disorder: results from the national epidemiologic survey on alcohol and related conditions (NESARC). J Clin Psychiatry. 2008;69(10):1606-1616.

37. Fredericks EM, Magee JC, Opipari-Arrigan L, Shieck V, Well A, Lopez MJ. Adherence and health-related quality of life in adolescent liver transplant recipients. Pediatr Transplant. 2008;12(3):289-299.

38. Wray J, Waters S, Radley-Smith R, Sensky T. Adherence in adolescents and young adults following heart or heart-lung transplantation. Pediatr Transplant. 2006;10(6):694-700.

39. Costanzo MR, Dipchand A, Starling R, et al. The international society of heart and lung transplantation guidelines for the care of heart transplant recipients. J Heart Lung Transplant. 2010;29(8):914-956.

How to cite this article: McCormick AD, Schumacher KR, Zamberlan M, et al. Generalized and specific anxiety in adolescents following heart transplant. Pediatr Transplant. 2020;24:e13647. https://doi.org/10.1111/petr.13647 